\title{
INTEGRATION FINANCIERE TEST ET EFFET SUR LA DIVERSIFICATION INTERNATIONALE
}

\author{
DR. AMARA Tijani ${ }^{1}$, SAMOUT Ammar ${ }^{2}$ \\ ${ }^{1}$ High Institute of Business Administration, University of Gafsa, Tunisia \\ tijaniamara@yahoo.fr \\ ${ }^{2}$ College of Economic Sciences and Management, University of Sfax, Tunisia \\ ammarbanq@yahoo.fr
}

\begin{abstract}
L'intégration des marchés financiers a fait l'objet de nombreuses études et travaux théoriques et empiriques qui tiennent leur importance du fait qu'en présence d'une intégration financière entre les marchés, la diversification internationale voit ses avantages se réduire voir s'éliminer complètement dans le cas d'une intégration totale. Ce ci étant, nous nous interrogeons a travers cette recherche dans quelle mesure les marchés financiers sont intégrés ? Et quel est l'impact du degré de l'intégration sur la diversification internationale des portefeuilles ?
\end{abstract}

Partant, l'objectif consiste à examiner l'intégration des marchés de capitaux, aussi bien au niveau des pays développés qu'au niveau des pays émergents. Pour répondre à cette problématique et atteindre l'objectif fixé, nous présentons dans la première partie de cette recherche une revue de la littérature sur les origines de l'intégration financière, la diversification internationale et l'intérêt des marches émergents et l'impact de l'intégration financière sur la diversification internationale. Dans la deuxième partie nous présentons la méthodologie de la recherche et les résultats dégagés.

\section{Key words:}

Marchés financiers, intégration, diversification, causalité, marchés émergents.

\section{Council for Innovative Research}

Peer Review Research Publishing System

Journal: International Journal of Management \& Information Technology

Vol. 8, No. 2

editor@cirworld.com

www.cirworld.com, member.cirworld.com 


\section{INTRODUCTION}

L'intégration de plusieurs pays dans les marchés financiers est l'un des faits stylisés marquant ces dernières décennies. Cette tendance a été stimulée par des conventions idéologiques selon lesquelles l'intégration financière internationale représente un optimum de premier rang et qu'elle est un symbole de réussite économique.

En outre, l'intégration des marchés financiers a fait l'objet de nombreuses études et travaux théoriques et empiriques qui tiennent leur importance du fait qu'en présence d'une intégration financière entre les marchés, la diversification internationale voit ses avantages se réduire voir s'éliminer complètement dans le cas d'une intégration totale.

Ainsi, l'intégration financière rend, d'un côté, la diversification internationale des portefeuilles plus efficace, et ce en facilitant le passage d'un marché à un autre et en améliorant l'efficience des marchés financiers. D'autre côté, l'intégration aurait augmenté les corrélations entre les marchés financiers nationaux, ce qui aurait réduit les bénéfices de la diversification.

L'objectif du travail, s'inscrit alors dans le contexte de l'ouverture financière et consiste à examiner l'intégration des marchés de capitaux, aussi bien au niveau des pays développés qu'au niveau des pays émergents. On s'attend que l'intégration des marchés mesurée d'une manière empirique nous permette de juger s'il est possible d'avoir un gain de diversification internationale ou non, c'est-à-dire, si les marchés sont intégrés, alors les gains potentiels seront faibles. En revanche, si les marchés sont segmentés alors la diversification internationale joue un rôle important. En effet, le concept de la diversification ainsi a été adopté par les investisseurs depuis longtemps comme étant un outil efficace pour l'amélioration du rendement de leurs portefeuilles. Elle permet d'une coté de réaliser des gains importants en affectant une partie du portefeuille à des actifs étrangers et d'autre coté de réduire son risque total.

La Théorie du portefeuille élaborée par Harry Markowitz et Arthur Roy, dans les années 1950, jette les bases de la théorie moderne du portefeuille et est à l'origine du concept de diversification des portefeuilles. Ces travaux prouvent que pour réduire le risque de portefeuille il est profitable d'adopter une stratégie de diversification des richesses.

Ainsi, Markowitz a montré qu'un comportement de diversification, consistant à investir sa richesse dans plusieurs actifs risqués, permet de réduire le risque global du portefeuille et de composer ce que l'on appelle le portefeuille efficient, c'est à dire le portefeuille qui offre une utilité maximale à l'investisseur grâce à sa composition optimale effectuée à partir de l'ensemble des titres disponibles sur le marché.

L'intégration du marché financier fait l'objet des études et de recherches académiques ; des bases de données et divers documentations se sont constituées, et beaucoup de chercheurs présentent leurs caractéristiques et les guident face à certaines contraintes. Mais rares sont ceux qui traitent les l'influence de l'intégration sur la diversification de portefeuille. C'est dans cette perspective que nous allons essayer d'introduire notre recherche et contribuer à élucider un certain nombre d'aspect de la question.

Nous tenterons dans cette recherche d'apporter une réponse à la question suivante : $\boldsymbol{A}$ quel point les marchés financiers sont intégrés? Et quel est l'impact du degré de l'intégration sur la diversification internationale des portefeuilles?

Pour répondre à cette problématique nous présentons dans la première partie de cette recherche une revue de la littérature sur les origines de l'intégration financière, la diversification internationale et l'intérêt des marches émergents et l'impact de l'intégration financière sur la diversification internationale. Dans la deuxième partie nous présentons la méthodologie de la recherche et les résultats dégagés.

\section{LES CONCEPTS FONDAMENTAUX DE L'INTEGRATION FINANCIERE}

\section{Les origines de l'intégration financière internationale}

On ne trouve pas dans la littérature empirique des marchés financiers, des travaux ayant pour but l'identification des facteurs de l'intégration financière. On trouve des travaux comme ceux de Bekaert et Harvey (1995,1997), Hardouvelis et al. (2002), Bhattacharya et Daouk (2002) et Adler et Qi (2003) qui ont arbitrairement retenu quelques variables financières et macroéconomiques dans la modélisation du degré d'intégration, mais n'ont pas formellement testé leur effet sur le degré d'intégration.

Parmi les causes de l'augmentation du degré d'intégration des marchés financiers on note le rôle des investisseurs internationaux, qui opèrent sur les différents marchés des capitaux, à accroître les co-mouvements des capitaux entre les différents marchés (Bekaert et Harvey (2002), Yi (2003)). Aussi, Kose, Pasard et Terrones (2003) montrent que l'augmentation des liens entre les marchés financiers n'est qu'une conséquence directe de l'intégration économique adoptée par la majorité des pays dans les années quatre-vingt-dix. Books, Forbes et Mody (2003) ont montré que les co-mouvements entre les marchés financiers ont augmenté durant les années 1990.

D'une autre coté, des nombreux travaux aussi bien théoriques qu'empiriques tel que les travaux de Bekaert et Harvey(1995,1997), Bhattacharya et Daouk(2002), Adler et Qi(2003) et Hardouvelis et al.(2006) ont montré qu'il existe plusieurs facteurs qui affectent le degré d'intégration financière. Parmi ces facteurs on note :

* Le développement du marché boursier: plus le marché devient développé et liquide, plus il attire les investisseurs étrangers et par conséquent agis positivement sur le degré d'intégration financière. 
* Le degré d'ouverture commerciale : plus le marché est ouvert au commerce extérieur, plus son degré d'intégration est élevé.

* La volatilité des taux de change : cette variable permet de décourager les transactions internationales et entraine la segmentation du marché.

* Le taux d'intérêt : cette variable affecte les mouvements internationaux de capitaux et permet d'agir sur le degré d'intégration financière.

* Le cout de capital : la baisse de ce cout augmente l'investissement et l'arbitrage internationaux et favorise l'intégration financière.

* Le taux d'inflation : plus ce taux est élevé, moins les investisseurs diversifient internationalement leurs portefeuilles ce qui favorise l'intégration financière.

\subsection{La diversification internationale et l'intérêt des marchés émergents}

Ces dernières années, les marchés financiers nationaux sont devenus plus intégrés suite aux mouvements de d'ouverture et de libéralisation entrepris par les différents gouvernements d'une part et aux innovations technologiques et financières d'autre part. Toutefois, malgré les évidences théoriques et empiriques des gains additionnels de la diversification internationale, les investisseurs ne diversifient pas suffisamment leurs portefeuilles.

En effet, le rythme accéléré des phénomènes de déréglementation et de libéralisation des marchés des capitaux a généré une forte intégration des économies dans le monde entier. De ce fait, les indices boursiers des marchés nationaux sont devenus de plus en plus corrélés entre eux ce qui affecte négativement avantages de la diversification internationale.

Dans ce contexte, on constate une diminution de l'intérêt de la diversification internationale sur les marchés développés suite à la forte intégration ce qui pousse les investisseurs à s'orienter vers les marchés émergents en raison de leur potentiel de croissance élevé et de leur segmentation aussi bien entre eux qu'avec les marchés développés. Donc quel est l'intérêt d'investir dans les marchés émergents ?

\subsubsection{Notion du pays émergents}

II semble indispensable avant toute chose, de définir ce que révèle un marché émergent. Tout d'abord aucune définition universelle n'est disponible jusqu'à présent. La définition la plus connue reste celle de la compagnie financière internationale (IFC), selon laquelle le terme 'marché émergents' se réfère en générale aux marchés d'actions qui sont en transition, et se caractérisent par une croissance de taille, d'activités et du niveau de sophistication. Du point de vue quantitatif, le statut 'émergent' est attribué à un marché d'actions s'il répond au moins à un des deux critères suivants:

- II appartient aux économies de faible et moyen revenu conformément à la classification de la banque mondiale

- $\quad$ La part de la capitalisation boursière en proportion de PNB est relativement faible.

\subsubsection{Intérêt de la diversification sur les pays émergents}

Beaucoup d'auteurs ont cherché à évaluer l'intérêt offert par les marchés d'actions émergents, dans le cadre d'une gestion internationale de portefeuille. En effet, eu égard à leur niveau de développement et compte tenu de leur cycle économique particulier, ces marchés devraient générer des potentiels de diversification par rapport aux marchés des pays développés.

Ces potentiels de diversification pourraient être d'autant plus importants que les économies développées ont tendance à être de plus en plus corrélées les unes aux autres, en raison de leur intégration croissante. A l'aide des "outils" développés par Markowitz (1952), et en posant l'hypothèse nulle que ces marchés s'inscrivent dans le paradigme "moyenne_variance", ces auteurs ont alors appliqué la théorie financière moderne afin d'apprécier le gain susceptible d'être obtenu par le biais de ces nouveaux actifs.

Le premier à avoir inclus un pays en voie de développement dans une stratégie internationale de diversification fut Grubel (1968) qui intégra l'Afrique du Sud dans un panel de 11 pays. Néanmoins, c'est avec Levy et Sarnat (1970), Lessard (1973) et surtout Errunza (1977) que l'impact des pays en développement sur la frontière d'efficience commença à être explicitement étudié.

Azman et al (2002) ont montré à travers des études portés sur les marchés de l'Indonésie, la Malaisie, la Thaïlande et du Singapour, que ces marchés ne sont pas parfaitement intégrés et par la suite il existe des opportunités de gain, sur le long terme, à partir de la diversification internationale sur ces marchés sud est asiatique.

En revanche, Bekaert, Harvey et $\mathrm{Ng}$ (2003) ont prouvé à travers le modèle d'évaluation des actifs financiers à double indice, l'existence d'une intégration régionale importante entre les marchés du sud-est asiatique. En outre, Jang et Sul (2002) ont confirmé l'existence d'une intégration importante au sein des marchés sud-est asiatique. Cela est expliqué par un accroissement significatif dans les co-mouvements des flux de capitaux dans la plus part de ces marchés.

A travers ses études portés sur les marchés des capitaux de l'Europe centrale (République Tchèque, Pologne et la Hongrie), Gilmore et Mc Manus (2002) ont signalé que ces marchés ne sont pas intégrés ni entre eux ni avec les 
marchés développés. Ce résultat implique l'existence de gains considérables de diversification internationale sur ces marchés.

Errunza (1977) a monté, en étudiant les rendements trimestriels hors dividendes des indices boursiers de 29 pays en dollar, de 1957 à 1971, que ces marchés ne sont pas parfaitement intégré, et il a trouvé faible corrélation du Venezuela, de l'Afrique du sud ou du Portugal avec les autres marchés que justifie leur admission dans les portefeuilles optimums, même si leur couple risque rendement respectif n'est pas parmi les meilleurs. .

Divecha, Drak et Stefek (1992) utilisent des données mensuelles converties en dollar, de février 1986 à mars 1991 pour 23 bourses des pays en voie de développement (inclut Jordanie, Malaisie, Nigeria, Pakistan, Turquie...) et 20 pays développé. Selon lui, la caractéristique primordiale de ces marchés réside dans leur faible corrélation entre eux, ce qui réduit leur risque global en tant que groupe, et dans leur faible corrélation avec les pays développés, ce qui donne lieu à des gains de diversification.

Les travaux de Sappenfield et Speidell (1992) ont été réalisés à partir de données trimestrielles de mars 1986 à mars 1991, converties en dollars, sur 18 marchés émergents et 18 marchés développés, ils notent une augmentation dans la corrélation entre les marchés développés en raison de l'impact mondial d'événements globaux tels le krach d'octobre 1987 ou l'invasion du Koweït en août 1990. Cette évolution génère un risque de corrélation, c'est à dire le risque qu'un portefeuille apparemment diversifié ne le soit plus dans le futur.

Ainsi, toutes ces études concluent l'intérêt des marchés d'actions émergents dans le cadre d'une gestion internationale de portefeuille, en raison de leur potentiel de diversification qui permet de réduire le niveau de risque.

\subsection{L'impact de l'intégration financière sur la diversification internationale des portefeuilles}

La diversification d'un portefeuille de titres ou d'actifs permet, soit de réduire le risque pour un niveau de rentabilité donné, soit d'améliorer la rentabilité pour un niveau de risque donné.

Cependant, ces dernières années, de nombreuses réformes ont été opérées dans les marchés financiers des différents pays. Ces réformes ont augmenté les interdépendances des marchés nationaux et amorcé le processus d'intégration financière internationale (Carrieri [2001] et Henry [2001]).

L'augmentation de l'intégration de façon accélérée aurait affecté les bénéfices attendus de la diversification internationale des portefeuilles. En effet, ces bénéfices dépendent des rentabilités, des volatilités et des corrélations des marchés nationaux. Ces dernières sont déterminées par les plusieurs facteurs de risque. Dans un marché parfaitement intégré, les structures des rentabilités dépendent des facteurs mondiaux de risque. Par contre, dans un marché strictement segmenté, ce sont les facteurs locaux qui jouent un rôle important. Au fur et à mesure que le degré d'intégration augmente, les actifs financiers deviennent de plus en plus sensibles aux facteurs internationaux de risque.

Les gains additionnels de la diversification internationale sont donc directement liés au niveau d'intégration financière.

Pour examiner les relations d'interdépendance entre les marchés développés et émergents et ses implications sur les gains de la diversification internationale on a eu recours à plusieurs approches.

Dans cette partie, on va étudier l'intégration financière et ses implications sur les gains des stratégies de diversification internationale de portefeuille à travers des études récentes menées sur le thème de l'intégration des marchés des capitaux en se basant sur plusieurs approches qui sont dont notamment le modèle d'évaluation des actifs financiers, les mesures basées sur les coefficients de corrélation et les mesures basées sur la méthode de cointégration

\section{Le modèle d'évaluation des actifs financiers}

Le littérature théorique et empirique montre que le modèle d'évaluation des actifs financiers à l'international et le modèle d'arbitrage international sont les deux modèles les plus utilisés pour l'estimation des valeurs des actifs financiers cotés sur divers marchés financiers et la validation de l'hypothèse de l'intégration de ces marchés. En effet, l'intégration des marchés financiers à travers ces modèles s'identifie par l'absence d'un écart entre les primes de risque pour des actifs financiers identiques échangés sur des places financières différentes.

Les modèles d'évaluation des actifs financiers internationaux permettent d'estimer les actifs et de tester l'hypothèse de l'intégration des marchés. Les marchés sont considérés intégrés si et seulement si les actifs de même risque, procurent le même rendement moyennant le taux de change.

De plus, lorsque les marchés des capitaux sont intégrés, les actifs financiers ayant les mêmes caractéristiques en terme de risque procurent des rendements espérés identiques qu'ils soient échangés ou non sur le même marché national.

Les modèles d'évaluation des actifs les plus connus sont le modèle d'évaluation des actifs financiers internationaux(MEDAFI) et le modèle d'arbitrage international(IAPT)

Bekaert, Harvey et $\mathrm{Ng}$ (2003) ont utilisé le modèle d'évaluation des actifs financiers à bêta conditionnel pour mesurer le degré d'intégration entre les marchés Européens, des Etats-Unis et de Royaume-Uni. Ils ont prouvé que les marchés Européens possèdent la corrélation conditionnelle la plus élevée avec les Etats-Unis ce qui confirme leur intégration aux marchés américains. 
De Santis et Gérard(1997) utilisent une spécification GARCH multivarié pour tester une version conditionnelle du MEDAF international. Leur étude porte sur les huit plus grands marchés (canada, japon, France, Allemagne, Italie, suisse, Grande-Bretagne et les Etats-Unis) et couvre la période 1970-1994. Les résultats de leur étude supportent le MEDAF international et donc l'hypothèse d'intégration des marchés financiers étudiés.

\section{Les mesures basées sur les coefficients de corrélation}

En basant sur les études qui portent sur l'intégration financière, on voie que le coefficient de corrélation des rendements entre les séries boursières représente un moyen pour examiner l'intégration des marchés financiers.

En effet, plus ce coefficient est proche de l'unité, plus l'hypothèse d'intégration est acceptée Dans ce cas, la diversification internationale n'est pas utile et il n'y a pas de gain important à attendre d'une telle stratégie étant donné que ces marchés évoluent d'une manière combinée.

Plusieurs économistes et financiers ont utilisés cette méthode, on note a titre d'indication Levy et Sarnat (1970) et Solnik (1974).

Les ouvrages dans ce domaine sont riches, comme par exemple, Grubel (1968), Levy et Sarnat (1970) et Lessard (1973) montrent que les faibles corrélations entre les marchés développés et émergents équité prouve que les avantages de la diversification internationale est considérable pour les investisseurs des pays industrialisés dans les marchés émergents.

D'autres études récentes document l'importance de la faible corrélation entre marchés développés et émergents pour générer des bénéfices substantiels de l'international diversification (Eun et Resnick, 1984; Errunza et Padmanabhan, 1988; Meric et Meric, 1989; Bailey et Stulz, 1990; Divecha et al. 1992; Phylaktis et Ravazzolo.

La conclusion finale de cette approche est que les avantages de la diversification du portefeuille international sont inversement proportionnels à la corrélation entre les rendements des marchés boursiers. La diversification internationale des gains baisse à mesure que les corrélations entre les rendements des titres de plus en plus positivent. Toutefois, l'existence de faibles corrélations entre les marchés nationaux peuvent fournir d'importants avantages de la diversification internationale

\section{Les mesures basées sur la méthode de co-intégration}

Le test de cointégration est connu surtout à travers les travaux de Granger(1986) afin de tester l'interdépendance entre les marchés financiers internationaux dans le contexte du non stationnarité des séries temporelles.

La cointégration se définie comme une relation d'équilibre à long terme entre les variables étudiées. Plusieurs études ont traité l'intégration des marchés des capitaux internationaux en utilisant la méthode de cointégration.

Plusieurs auteurs ont utilisé les techniques de cointégration pour montrer l'existence des liens et à long terme de co-mouvements entre les marchés développés et émergents. Ils analysent leurs effets sur les avantages de la diversification internationale pour l'investissement dans les marchés émergents.

Masih et al qui ont appliqué la technique de cointégration sur une série des indices boursiers journaliers de quatre pays d'OCDE et quatre pays asiatiques, leurs résultats confirment également l'hypothèse de l'intégration.

Brooks, Forbes et Mody (2003) montrent que les co-mouvements entre les marchés boursiers augmenté durant les années 90 et ceci s'expliquent par un accroissement du degré d'intégration entre les marchés en question

\section{Méthodologie de la recherche et présentation des résultats}

Pour examiner les relations d'interdépendance entre les marchés développés et émergents et ses implications sur les gains de la diversification internationale on a eu recours à l'approche de cointégration de Johansen (1988). Cette dernière permet de détecter l'existence d'un

co-mouvement entre les indices boursiers des différents marchés nationaux sur le long terme.

Les bases de données utilisées dans cette étude sont des données journalières des prix des indices boursiers de 5 marchés développés et 5 marchés émergents. Ils ont été extraits de la base du site "Yahoo Finance et économiste 》. Ces indices couvrent la période allant du janvier 2004 jusqu'au juin 2009, ce qui nous donne 1696 observations par marché. L'échantillon est comme suit :

\begin{tabular}{|c|c|}
\hline Marchés développés & Marchés émergents \\
\hline - Amérique du nord : Etats-Unis, & - Amérique latine : Argentine, Mexique, \\
\hline - Europe : France, Allemagne, Royaume Unis, & - Asie : Chine, Malaisie \\
\hline - Asie : Japon, & - Afrique : Egypte \\
\hline
\end{tabular}




\section{Analyse descriptive de l'échantillon}

Le tableau (1) et (2) ci-dessous regroupent les statistiques descriptives des rendements des indices boursiers des marchés développés et émergents retenus dans l'échantillon qui se décompose de 1696 observations.

Tableau.1. Statistiques descriptives des rendements des indices boursiers des marchés développés $\left(\right.$ Rendement $\left.t=\operatorname{Ln}\left(P_{t+1} / P_{t}\right)\right)$

\begin{tabular}{|c|c|c|c|c|c|}
\hline & ETATS UNIS & R.U & FRANCE & ALLEMAGNE & JAPON \\
\hline Moyenne & $-0,000021$ & 0,000191 & 0,000194 & 0,000205 & 0,0000631 \\
\hline Médian & 0,0000217 & 0,000331 & 0,000602 & 0,000847 & 0,000000 \\
\hline Maximum & 0,056104 & 0,052560 & 0,059568 & 0,068889 & 0,057712 \\
\hline Minimum & $-0,052244$ & $-0,052735$ & $-0,063355$ & $-0,077757$ & $-0,071582$ \\
\hline Ecart type & 0,010775 & 0,011083 & 0,013302 & 0,015074 & 0,013427 \\
\hline Skewness & 0,137767 & $-0,253787$ & $-0,175461$ & $-0,186547$ & $-0,161563$ \\
\hline Kurtosis & 5,974201 & 5,812756 & 5,442188 & 5,531503 & 4,656866 \\
\hline Jarque-Bera & 630,4731 & 577,2920 & 430,1781 & 462,7047 & 201,3729 \\
\hline Probabilité & 0,000000 & 0,000000 & 0,000000 & 0,000000 & 0,000000 \\
\hline Observations & 1696 & 1696 & 1696 & 1696 & 1696 \\
\hline
\end{tabular}

Ce tableau illustre les statistiques descriptives des cinq séries d'étude. On constate d'après ce tableau que pour les marchés développés, les rendements moyens les plus élevés sont attribués à la bourse d'Allemagne 0,020\% et la France avec $0.0194 \%$ tandis que celui le plus faible est enregistré aux Etats-Unis ; soit $-0,002 \%$. Le rendement maximal varie de $6,88 \%$ au Allemagne à $5,25 \%$ en Royaume Unis alors que le rendement minimal fluctue entre $7,77 \%$ en Allemagne et $-5,22 \%$ aux Etats-Unis. En terme de risque, la bourse des Etas unis possède le risque le moins élevé ; soit, $10.775 \%$ alors que celui le plus élevé est marqué à la bourse de l'Allemagne avec un écart type de $15.074 \%$.

On constate en premier lieu le coefficient de Kurtosis est très faible, c'est à dire inférieur à 3 pour tous les variables étudies (valeur du coefficient de Kurtosis pour la loi normale). En second lieu, le coefficient de Skewness est différent de zéro (valeur théorique du coefficient de Skewness pour une loi normale) et négatif sauf pour le cas des Etats Unis.

Le coefficient de Skewness indique en effet que la distribution est étalée vers la gauche. Par conséquent, l'hypothèse de normalité n'est pas vérifié et le test de Jarque-Bera confirme bien ce résultat et rejette significativement la distribution normale des rendements des indices boursiers pour tous les marchés formant l'échantillon.

Tableau.2. Statistiques descriptives des rendements des indices boursiers des marchés émergents

\begin{tabular}{|c|c|c|c|c|c|}
\hline & ARGENTINE & CHINE & EGYPTE & MALAISIE & Mexique \\
\hline Moyenne & 0,000446 & 0,000386 & 0,001040 & 0,000413 & 0,000731 \\
\hline Médian & 0,000385 & 0,000329 & 0,000000 & 0,000000 & 0,001070 \\
\hline Maximum & 0,163412 & 0,069609 & 0,092859 & 0,049610 & 0,073002 \\
\hline Minimum & $-0,336472$ & $-0,099276$ & $-0,090046$ & $-0,069725$ & $-0,075113$ \\
\hline Ecart type & 0,025453 & 0,016473 & 0,017041 & 0,009078 & 0,014401 \\
\hline
\end{tabular}




\begin{tabular}{|c|c|c|c|c|c|}
\hline Skewness & $-1,850592$ & $-0,357656$ & 0,010399 & $-0,541144$ & $-0,148834$ \\
\hline Kurtosis & 30,46395 & 5,915317 & 7,033792 & 9,771263 & 5,496460 \\
\hline Jarque-Bera & 54269,71 & 636,7593 & 1149,882 & 3322,842 & 446,6781 \\
\hline Probabilité & 0,000000 & 0,000000 & 0,000000 & 0,000000 & 0,000000 \\
\hline Observations & 1696 & 1696 & 1696 & 1696 & 1696 \\
\hline
\end{tabular}

En ce qui concerne les marchés émergents (tableau2), l'Egypte possède le rendement moyen le plus élevé ; soit $0,104 \%$, alors que celui le plus faible correspond à la bourse de Chine 0,038\%. Le rendement maximal varie de $16,34 \%$ en Argentine à $9,28 \%$ en Egypte tandis que le rendement minimal varie de $-33,64 \%$ à l'Argentine à $4,96 \%$ au Malaisie.

En terme de risque, la bourse de Malaisie possède le risque le moins élevé ; soit, $0,90 \%$ alors que celui le plus élevé est marqué à la bourse de l'Argentine avec un écart type de 2,54\%.

Les valeurs de la statistique de Kurtosis indiquent que les séries présentent un caractère épais. Les coefficients de Skewness indiquent que la distribution est asymétrique à gauche et rejettent la distribution normale pour la majorité des séries. Par conséquent, l'hypothèse de normalité n'est pas vérifié et le test de Jarque-Bera confirme bien ce résultat et rejette significativement la distribution normale des rendements des indices boursiers pour tous les marchés formant l'échantillon.

\section{Etude des corrélations des rendements}

L'étude des coefficients de corrélation entre les rendements des indices boursiers est la technique la plus simple pour expliquer l'intégration des marchés financiers. En effet, plus ce coefficient est proche de l'unité, plus l'hypothèse d'intégration est acceptée étant donné qu'un tel résultat signifie que les marchés incorporent l'information de manière identique. Dans ce cas, la diversification internationale n'est pas utile et il n'y a pas de gain important à attendre d'une telle stratégie étant donné que ces marchés évoluent d'une manière combinée. Cette méthode a été utilisée par Levy et Sarnat (1970) et Solnik (1974) en vue d'identifier les bienfaits à court terme de la diversification internationale. Les tableaux (3) et (4) présentent les coefficients de corrélation entre les rendements des indices boursiers des marchés développés.

Tableau.3. Les corrélations des rendements entre les indices boursiers des marchés développés et
\begin{tabular}{|c|c|c|c|c|c|}
\hline & Argentine & Chine & Malaisie & Mexique & Egypte \\
\hline Etats-Unis & 0.199990 & 0.117221 & 0.012136 & 0.575003 & -0.025451 \\
\hline Royaume Unis & 0.199973 & 0.233428 & 0.111306 & 0.437825 & -0.006080 \\
\hline France & 0.198311 & 0.228802 & 0.107472 & 0.474218 & -0.018482 \\
\hline Allemagne & 0.183371 & 0.200253 & 0.072218 & 0.503687 & -0.017169 \\
\hline Japon & 0.091131 & 0.420451 & 0.248127 & 0.155539 & 0.089430 \\
\hline
\end{tabular}

Les coefficients de corrélation entre les rendements des marchés développés et émergents sont très faibles et parfois négatifs. Cela montre bien que les marchés émergents sont très faiblement corrélés avec les marchés développés comme le montre le tableau (3). Le niveau de corrélation le plus élevé est enregistré entre les Etats-Unis et le Mexique, soit $57,50 \%$ suivie du marché d'Allemagne et de Mexique 50,36\%. Cependant, le coefficient de corrélation le plus faible est de $-2,54 \%$ entre les Etats unis et l'Egypte. Ainsi, par rapport aux marchés développés qui présentent une forte corrélation entre eux impliquant ainsi leur forte intégration, les marchés émergents présentent une faible relation de dépendance aussi bien entre eux qu'avec les marchés développés.

Cette indépendance entre les marchés développés et émergents implique une segmentation. Cette segmentation permet de réaliser des gains énormes de diversification internationale sur ces marchés émergents. En effet, eu égard à leur niveau de développement et compte tenu de leur cycle économique particulier, ces marchés émergents devraient générer des potentiels de la diversification par rapport aux marchés des pays développés.

Nos résultats prouvent que les marchés développés sont intégrés entre eux alors que les marchés émergents sont segmentés aussi bien entre eux qu'avec les marchés développés. Ces conclusions sont conformes avec la majorité des études menées sur le thème de l'intégration des marchés financiers tel que celle de Gilmore et McManus (2002) 
et Bekaert, Harvey et $\mathrm{Ng}$ (2003). Cela implique que ces marchés émergents représentent encore une source importante de diversification internationale de portefeuille, malgré l'accroissement de l'intégration financière qui favorise l'augmentation des corrélations entre les marchés nationaux ce qui réduirait les gains des stratégies de diversification internationale et il faut tenir compte aussi les transmissions des chocs surtout suite aux nombreuses crises qui ont frappé les marchés financiers ces dernières années (La crise de Subprime).

\section{Etude de la cointégration}

\subsection{Test de racine unitaire des séries : test ADF}

\subsubsection{Test de racine unitaire des séries : pays émergents}

\section{a. Stationnarité des séries en niveau}

On test ici les hypothèses:

$\mathrm{H}^{0}$ : la série en niveau est non stationnaire

$\mathrm{H}^{1}$ : la série en niveau est stationnaire

L'application de test de racine unitaire des séries en niveau nous permet de dresser le tableau suivant :

Tableau 4. Stationnarité en niveau : pays émergents

\begin{tabular}{|c|c|c|c|c|c|}
\hline Pays & France & Germany & japon & R.U & USA \\
\hline T. Statistique & 1.476123 & 1.609054 & 0.720213 & 2.136614 & 2.633606 \\
\hline $\begin{array}{c}\text { Valeurs critiques (seuils 1\%, 5\% } \\
\text { et 10\%) }\end{array}$ & -2.566326 & -2.566574 & -3.434009 & -2.566326 & -2.566326 \\
& -1.616574 & -1.616574 & -1.616574 & -1.616574 & -1.941010 \\
& & & & & -1.616574 \\
\hline Probabilité & 0,9658 & 0,9742 & 0.9926 & 0,9926 & 0.9982 \\
\hline Stationnarité & non & non & non & non & non \\
\hline
\end{tabular}

Le tableau(4) relatif au test ADF au sein de la zone émergente fait ressortir une valeur de t statistique inférieur en valeur absolue aux valeurs critiques pour les trois seuils (1\%,5\% et $10 \%)$. Les indices boursiers de ces pays sont donc non stationnaires. En conséquence à cette non stationnarité, nous passons du test niveau à la première différenciation (variation de l'indice).

\section{b. Stationnarité des séries différenciées}

On test ici l'hypothèse :

$\mathrm{H}^{0}$ : la série en différence première est non stationnaire

$\mathrm{H}^{1}$ : la série en différence première est stationnaire

L'application de test de racine unitaire des séries différenciées nous permet de dresser le tableau suivant :

Tableau 5. Stationnarité des séries différenciées : pays émergents

\begin{tabular}{|c|c|c|c|c|c|}
\hline Pays & Argentine & Chine & Egypt. & Malaisie & Mexico \\
\hline T. Statistique & -40.53294 & -37.99137 & -19.83045 & -32.91454 & -36.2636 \\
\hline Valeurs critiques (seuils 1\%,5\% et & -2.566327 & -2.566574 & -3.434009 & -2.566574 & 2.566574 \\
$\mathbf{1 0 \% )}$ & -1.941010 & -1.941010 & -2.863043 & -1.941010 & -1.941010 \\
& -1.616574 & -1.616574 & -2.567618 & -1.616574 & -1.616574 \\
\hline Probabilité & 0.0000 & 0.0000 & 0.0000 & 0.0000 & 0.0000 \\
\hline Stationnarité & oui & oui & oui & oui & oui \\
\hline
\end{tabular}


Le tableau (5) relatif au test ADF au sein de la zone émergente nous indique que les t statistiques des pays sont largement supérieurs en valeur absolue aux différents seuils critiques déjà énumérés. Nous concluons que les indices relatifs à ces pays sont intégrés d'ordre (1) ou I(1).

\subsubsection{Test de racine unitaire des séries: Pays développés}

\section{a. Stationnarité des séries en niveau}

On test ici les hypothèses :

$\mathrm{H}^{0}$ : la série en niveau est non stationnaire

$\mathrm{H}^{1}$ : la série en niveau est stationnaire

L'application de test de racine unitaire des séries en niveau nous permet de dresser le tableau suivant :

Tableau 6. Stationnarité en niveau : pays développés

\begin{tabular}{|c|c|c|c|c|c|}
\hline Pays & France & Germany & Japon & R.U & USA \\
\hline T. Statistique & 0.954604 & 0.980872 & 0.083750 & 0.154461 & -1.7536743 \\
\hline Valeurs critiques (seuils 1\%, 5\% et & -2.566326 & -2.566574 & -2.566327 & -3.434004 & -3.434004 \\
$\mathbf{1 0 \% )}$ & -1.941010 & -1.941010 & -1.1941010 & -2.863041 & -2.863041 \\
& -1.616574 & -1.616574 & -1.616574 & -2.567616 & -2.567616 \\
\hline probabilité & 0.9102 & 0.9141 & 0.7091 & 0.9696 & 0.4009 \\
\hline stationnarité & non & non & non & non & non \\
\hline
\end{tabular}

Le tableau(6)relatif au test ADF au sein de la zone développée fait ressortir une valeur de t statistique inférieur en valeur absolue aux valeurs critiques pour les trois seuils $(1 \%, 5 \%$ et $10 \%)$. Les indices boursiers de ces pays sont donc non stationnaires. En conséquence à cette non stationnarité, nous passons du test niveau à la première différenciation (variation de l'indice).

\section{b. Stationnarité des séries différenciées}

On test ici l'hypothèse :

$\mathrm{H}^{0}$ : la série en différence première est non stationnaire

$\mathrm{H}^{1}$ : la série en différence première est stationnaire

L'application de test de racine unitaire nous permet de dresser ce tableau :

Tableau 7. Stationnarité des séries différenciées : pays développés

\begin{tabular}{|c|c|c|c|c|c|}
\hline Années & France & Germany & Japon & R.U & USA \\
\hline T. Statistique & -40.91449 & 40.84984 & -40.80654 & -42.20899 & -42.149421 \\
\hline Valeurs critiques (seuils 1\%, 5\% et & -2.566327 & -2.566574 & -2.566327 & -3.434004 & -3.434004 \\
$\mathbf{1 0 \% )}$ & -1.941010 & -1.941010 & -1.1941010 & -2.863041 & -2.863041 \\
\hline probabilité & -1.616574 & -1.616574 & -1.616574 & -2.567616 & -2.567616 \\
\hline stationnarité & 0.0000 & 0.0000 & 0.0000 & 0.0000 & 0.0000 \\
\hline oui & oui & oui & oui & oui \\
\hline
\end{tabular}

Le tableau(7) relatif au test ADF au sein de la zone développée nous indique que les t statistiques des pays sont largement supérieurs en valeur absolue aux différents seuils critiques déjà énumérés. Nous concluons que les indices relatifs à ces pays sont intégrés d'ordre (1) ou I(1).

Les différents indices ont le même ordre d'intégration, En effet, selon les tests précédents, nous avons explicité que ces séries sont intégrées d'ordre 1 ou I(1),

Pour estimer la cointégration des séries sur le long terme, nous pouvons procéder à un test de cointégration multi varié, ou encore appelé test de Johansen (1988) et/ ou à un test de cointégration bi varié d'Engle et Granger (1987) ; et sur le court terme, nous procéderons au test du VECM (Modèle de Vecteur à Correction d'Erreur).

\subsection{La détermination du nombre de retard}




\subsubsection{La détermination du nombre de retard: les marchés développés}

Pour l'ensemble des marchés développés, les critères AIC et SC sont minimaux pour un $P=3$ et donc le modèle VAR à retenir est d'ordre 3 .

Tableau 8 Détermination du nombre de retard $(p)$ : pays développées

\begin{tabular}{|c|c|c|c|c|}
\hline Critères & VAR(1) & VAR(2) & VAR(3) & VAR(4) \\
\hline Akaike & 7.621856 & 7.537765 & 7.522322 & 7.523268 \\
\hline Schwarz & 7.641088 & 7.573039 & 7.575654 & 7.590675 \\
\hline
\end{tabular}

\subsubsection{La détermination du nombre de retard: les marchés émergents}

Pour l'ensemble des marchés émergents, les critères AIC et SC sont minimaux pour un $\mathrm{P}=2$ et donc le modèle VAR à retenir est d'ordre 2 .

Tableau 9 Détermination du nombre de retard $(p)$ : pays émergents

\begin{tabular}{|c|c|c|c|c|}
\hline Critères & $\operatorname{VAR}(1)$ & $\operatorname{VAR}(2)$ & $\operatorname{VAR}(3)$ & $\operatorname{VAR}(4)$ \\
\hline Akaike & 1.02716 & 0.904839 & 0.909587 & 0.910308 \\
\hline Schwarz & 1.039947 & 0.940113 & 0.960920 & 0.977715 \\
\hline
\end{tabular}

La détermination du nombre de retard $(P)$ du modèle vectoriel autorégressif VAR $(P)$ est une étape importante dans notre étude empirique sur l'intégration des marchés. Pour le cas des marchés développés, on a un VAR d'ordre 3 puisque les critères AIC (Akaike information criterion) et SC (Schwarz criterion) sont minimaux pour un $\mathrm{P}=3$ (tableau 10).Pour ce qui concerne les pays émergents on a un VAR d'ordre 2 puisque les critères sont nominaux pour un $P=$ 2, (Tableau 9)

\subsection{Test de cointégration}

Les hypothèses du test sont :

$\mathrm{H}^{0}$ : Présence au moins d'une relation de cointégration

$\mathrm{H}^{1}$ : Absence de relation de cointégration entre les séries

\subsubsection{Test de cointégration : les marchés développés}

L'application du test de Johansen nous permet de dresser le tableau ci-dessous :

Tableau 10. Test de Co-intégration multi-variée de Johansen : Pays développés

\begin{tabular}{|c|c|c|c|}
\hline $\mathrm{N}^{\circ}$ de relation de cointégration & Valeur propre & Trace statistique & Valeur critique (5\%) \\
\hline Aucun* $^{*}$ & 0.031470 & 127.1427 & 88.80380 \\
\hline Au plus 1* & 0.015889 & 73.00827 & 63.87610 \\
\hline Au plus 2* & 0.012465 & 45.89230 & 0.024528 \\
\hline Au plus 3 & 0.009623 & 24.65830 & 25.87211 \\
\hline Au plus 4 & 0.004883 & 8.287718 & 12.51798 \\
\hline
\end{tabular}

* indique qu'on doit rejeter $\mathrm{H}^{1}$ et passer à la deuxième itération.

Le test de cointégration multivarié de Johansen entre les marchés développés montre l'existence de trois relations de cointégration. Ces résultats montrent que ces marchés sont intégrés entre eux sur le long terme. En effet, la présence de cette relation de cointégration entre ces marchés explique leurs convergences vers un certain niveau d'équilibre stable de long terme. Par la suite, la présence de cette relation de cointégration traduit donc de l'intégration de ces marchés développés ce qui affecte négativement les gains de la diversification internationale.

\subsubsection{Le test de cointégration : les marchés émergents}


Tableau 11. Test de Co-intégration multi-variée de Johansen : Pays émergents

\begin{tabular}{|c|c|c|c|}
\hline$N^{\circ}$ de relation de cointégration & Valeur propre & Trace statistique & Valeur critique (5\%) \\
\hline Aucun* & 0.024664 & 96.30380 & 88.80380 \\
\hline Au plus 1 & 0.014183 & 53.99990 & 93.87610 \\
\hline Au plus 2 & 0.010389 & 29.80261 & 42.91525 \\
\hline Au plus 3 & 0.005457 & 12.11128 & 25.87211 \\
\hline Au plus 4 & 0.001676 & 2.841974 & 12.51798 \\
\hline
\end{tabular}

Le test de cointégration multivarié de Johansen entre les marchés émergents montre l'existence d'une seule relation de cointégration.

Pour étudier les mouvements de court terme on a eu recours au modèle VECM (Vecteur Error Correction Model). D'après les résultats obtenu dans le tableau (12) on remarque que les coefficients d'ajustements vers l'équilibre ne sont pas négatifs ce qui implique que le VECM n'est pas valide et par conséquent ces marchés ne sont pas intégrés sur le court terme.

Ces résultats impliquent que ces marchés émergents peuvent encore offrir des sources et des opportunités de gains de diversification internationale de portefeuille surtout pour les investisseurs des pays développés.

L'application du modèle de VECM nous permet de dresser le tableau ci-dessous :

Tableau 12. Modèle VECM : Pays émergents

\begin{tabular}{|c|c|c|c|c|c|}
\hline Conditions & Argentine & Chine & Egypt. & Malaisie & Mexico \\
\hline Significativité & $-0.020314^{\star}$ & $6.61 \mathrm{E}-05$ & 0.001341 & $-0.001039^{*}$ & $-0.00086^{*}$ \\
\hline Force de rappel $(<0)$ & négative & positive & positive & négative & négative \\
\hline
\end{tabular}

${ }^{*}$ le coefficient est significatif au seuil de $5 \%$

\subsubsection{Test de cointégration : marchés développés}

Tableau 13. Test de Co-intégration multi-variée de Johansen : Pays émergents-pays développés

\begin{tabular}{|c|c|c|c|}
\hline$N^{\circ}$ de relation de cointégration & Valeur propre & Trace statistique & $\begin{array}{c}\text { Valeur critique } \\
(5 \%)\end{array}$ \\
\hline Aucun* $^{*}$ & 0.048941 & 324.9026 & 273.1889 \\
\hline Au plus 1* & 0.037650 & 239.8761 & 228.2979 \\
\hline Au plus 2 & 0.026941 & 174.8649 & 187.4701 \\
\hline Au plus 3 & 0.018810 & 92.08150 & 117.7082 \\
\hline Au plus 4 & 0.011328 & 59.51393 & 88.80380 \\
\hline Au plus 5 & 0.011328 & 59.51393 & 88.80380 \\
\hline Au plus 6 & 0.008792 & 40.91461 & 93.87610 \\
\hline Au plus 7 & 0.007656 & 25.65500 & 42.91525 \\
\hline Au plus 8 & 0.005001 & 12.63499 & 25.87211 \\
\hline Au plus 9 & 0.002442 & 4.141812 & 12.51798 \\
\hline
\end{tabular}

Le tableau ci-dessus (tableau 13) présente la cointégration entre les marchés émergents et les marchés développés, il montre qu'il existe une relation de cointégration 


\section{La causalité : preuve de l'intégration}

L'idée de causalité en économie est une idée ancienne, fruit d'économistes et financiers qui trouvent dans les techniques économétriques un moyen d'être établie et testée. Le sens de la causalité économique est un élément essentiel pour élaborer une politique économique ou pour effectuer des prévisions.

Les hypothèses du test sont :

$H^{0}$ : L'indice $X$ ne cause pas l'autre indice $Y$

$H^{1}$ : L'indice $X$ cause l'autre indice $Y$

\subsection{La causalité entre les pays émergents}

L'application du test de causalité aux séries des indices boursiers des marchés émergents nous permet de dresser le tableau ci-dessous :

Tableau 14: causalité des pays émergents

\begin{tabular}{|c|c|c|}
\hline Sens de relation de causalité & probabilité & Décision de causalité \\
\hline Chine $\quad \rightarrow$ argentine & 0.176 & NON \\
\hline Argentine $\rightarrow$ chine & 0.0536 & NON \\
\hline Egypt. $\quad \rightarrow$ argentine & 0.0762 & NON \\
\hline Argentine $\rightarrow$ égypt. & 2.8E-06 & OUI \\
\hline Malaisie $\rightarrow$ argentine & 0.09448 & NON \\
\hline Argentine $\rightarrow$ Malaisie & 1.3E-10 & OUI \\
\hline Mexico $\rightarrow$ argentine & 0.01344 & OUI \\
\hline Argentine $\rightarrow$ mexico & 0.12352 & NON \\
\hline Egypt. $\quad \rightarrow$ chine & 0.0547 & NON \\
\hline Chine $\rightarrow$ égypt. & 0.1723 & NON \\
\hline Malaisie $\rightarrow$ chine & 0.80249 & NON \\
\hline Chine $\quad \rightarrow$ Malaisie & 0.00980 & OUI \\
\hline Mexico $\rightarrow$ chine & $7.2 E-39$ & OUI \\
\hline Chine $\rightarrow$ mexico & 0.08903 & NON \\
\hline Malaisie $\rightarrow$ égypt. & 3.8E-05 & OUI \\
\hline Egypt. $\quad \rightarrow$ Malaisie & 0.38015 & NON \\
\hline Mexico $\rightarrow$ égypt. & $4.5 E-14$ & OUI \\
\hline Égypt. $\rightarrow$ mexico & 0.24156 & NON \\
\hline Mexico $\rightarrow$ Malaisie & 0.0786 & NON \\
\hline Malaisie $\rightarrow$ mexico & 0.18866 & NON \\
\hline
\end{tabular}

La détermination du test de causalité au sens de Granger a montré que les couples des marchés - Chine, Malaisie.Mexique, Chine présentent des relations bidirectionnelles sur le long terme donc tout choc se répercutera sur chaque pays. Pour les couples -Egypt. Argentine- Malaisie argentine- Mexico argentine- Mexico égypt.- Mexico chine- 
Malaisie chine- Argentine Mexique. On remarque d'après les probabilités liées à la statistique de ficher au seuil $5 \%$ qu'il existe des relations de causalité unidirectionnelle.

Ainsi, les chocs qui affectent un marché donné vont influencer très significativement les mouvements de l'autre marché. En revanche, un choc sur ce dernier marché ne se transmet pas sur le premier marché. Plusieurs facteurs expliquent ces résultats tels que la crise asiatique, les mouvements et les liens entre ces pays et les bouleversements de la scène financière mondiale.

D'autre part notre étude montre l'existence des relations de non causalité entre les couples- Mexico Malaisie- Chine Argentine-Argentine Mexique. Ce résultat traduit la segmentation entre ces marché ce qui constitue un milieu favorable pour bénéficier des gains de diversification.

\subsection{La Causalité entre les pays développés}

Les hypothèses du test sont :

$\mathrm{H}^{0}$ : L'indice $X$ ne cause pas l'autre indice $Y$

$\mathrm{H}^{1}$ : L'indice $\mathrm{X}$ cause l'autre indice $\mathrm{Y}$

L'application du test de causalité aux séries des indices boursiers des marchés développés nous permet de dresser le tableau ci-dessous :

Tableau 15: Causalité des pays développés

\begin{tabular}{|c|c|c|}
\hline Sens de relation de causalité & Probabilité & Décision de causalité \\
\hline Germany $\rightarrow$ France & 7.0E-09 & OUI \\
\hline France $\rightarrow$ Germany & 0.00472 & OUI \\
\hline Japon $\rightarrow$ France & 0.52889 & NON \\
\hline France $\rightarrow$ Japon & $1.6 \mathrm{E}-38$ & OUI \\
\hline Royaume uni $\rightarrow$ France & 0.10152 & NON \\
\hline France $\rightarrow$ R.U & 0.01446 & OUI \\
\hline USA $\rightarrow$ France & $5.2 \mathrm{E}-37$ & OUI \\
\hline France $\rightarrow$ USA & 0.00510 & OUI \\
\hline Japon $\rightarrow$ Germany & 0.85916 & NON \\
\hline Germany $\rightarrow$ Japon & $1.4 \mathrm{E}-41$ & OUI \\
\hline R.U $\rightarrow$ Germany & 0.00541 & OUI \\
\hline Germany $\rightarrow$ R.U & 3.7E-07 & OUI \\
\hline USA $\rightarrow$ Germany & $6.2 \mathrm{E}-15$ & OUI \\
\hline Germany $\rightarrow$ USA & 0.01209 & OUI \\
\hline R.U $\rightarrow$ Japon & $2.7 \mathrm{E}-27$ & OUI \\
\hline Japon $\rightarrow$ R.U & 0.85629 & NON \\
\hline USA $\rightarrow$ japon & 3.7E-42 & OUI \\
\hline Japon $\rightarrow$ USA & 0.07056 & NON \\
\hline USA $\rightarrow$ R.U & $5.4 \mathrm{E}-34$ & OUI \\
\hline R.U $\rightarrow$ USA & 0.00415 & NON \\
\hline
\end{tabular}

La détermination du test de causalité au sens de Granger a montré que les couples des marchés : Germany France USA France - Royaume uni Germany - USA Germany présentent des relations bidirectionnelles sur le long terme donc tout choc se répercutera sur chaque pays. Aussi, le recours au test de causalité de Granger nous permet d'identifier le sens de causalité entre ces marchés cointégrés.

D‘après le tableau (15), nous constatons pour les couples des marchés -Japon France-

Royaume-Unis France - Japon Germany - Royaume uni - Japon-USA Royaume -uni qu'il existe des relations de causalité unidirectionnelle du couple de marché. 
Par exemple pour le couple Japon- USA un choc sur le marché américain va influencer sur le marché japonais. En revanche un choc sur le marché japonais n'affecte pas le marché américain. Donc le test de causalité confirme bien la relation de court terme

\section{CONCLUSION}

Avec la levée progressive de différentes barrières à l'investissement international et la globalisation financière, les investisseurs se sont mis à diversifier leurs placements. Ainsi, pour tout portefeuille la gestion de risque a deux objectifs, améliorer la performance financière et prévenir toute perte non supportable. La diversification internationale permet aux acteurs d'atteindre ces deux objectifs simultanément. Elle permet d'augmenter le rendement et de diminuer le risque de portefeuille.

Nous avons préféré dans un premier temps de passer en revue une profonde analyse sur l'intégration financière. Ensuite nous avant exposé la diversification internationale comme étant la meilleure stratégie permettant de réduire le risque de portefeuille et on a montré théoriquement l'impact de l'intégration financière sur la diversification a travers plusieurs approches tel que la corrélation entre les marchés financiers et la cointégration.

Enfin, nous avons d'abord testé les bénéfices de la diversification internationale. Les résultats montrent que les marché développés sont fortement corrélé ( 0,85 pour la France et l'Allemagne) donc investir exclusivement dans des titres des marché développés n'est pas bénéfique. Par ailleurs, les corrélations entre les marchés développés et émergents et celles entre les marchés émergent sont faibles et même négatives (Egypte et l'argentine -0,017). Par conséquent, bénéficier de cette stratégie il est intéressant de combiner des actifs financiers des marchés développés et des marchés émergents.

De plus en se basant sur le test de la cointégration les résultats montrent que dans la plupart des couples des marchés émergents des pays étudiés on trouve une absence de la cointégration cela entraine une importance des gains sur ces marché et d'autre part en ce qui concerne les marché développés on a trouvé l'existence des relations de cointégration ce qui se traduit par une baisse des gains de la diversification.

Par ailleurs les résultats de test de causalité montre bien l'existence des relations de non causalité entre les couplesMexico Malaisie- Chine Argentine-Argentine Mexique. Ce résultat traduit la segmentation entre ces marché ce qui constitue un milieu favorable pour bénéficier des gains de diversification.

Néanmoins, notre travail de recherche présente certaines limites : la première concerne les techniques employées pour tester les bénéfices de la diversification internationale. La deuxième limite concerne le nombre de pays de notre échantillon qui est faible et le non prise en compte de l'effet des taux de change sur les variations des rendements des indices boursiers. A la lumière de ces limites, des recherches futures utilisent d'autres techniques de mesure des bénéfices de la diversification internationales (modèles d'évaluation des actifs financiers) afin permettre aux gérants de portefeuilles de mieux évaluer le risque et de mieux sélectionner les pays et les titres dans lesquels il faut investir.

D'autre part, il fut rapidement constaté que les investisseurs contrairement aux attentes de la théorie, ne diversifient pas suffisamment leurs portefeuilles, et qu'ils ont forte préférence pour les actifs domestique. Cela s'explique par la préférence local dite aussi biais domestique, qui l'on va développerai dans des d'autres travaux et on va essayeras d'expliquer comment la crise représente un bais domestique

\section{BIBLIOGRAPHY}

[1] ADLER M. ET DUMAS B., (1983) «International Portfolio Choice and Corporation Finance: a Synthesis », journal of finance, vol. 38, pp. 925-984.

[2] AHEARNE G, WILIAM L. et FRANCIS E. (2001), «Information Costs and Home Bias: An Analysis of US Holdings of Foreign Equities», Board of Governos of the Federal Reserve System: International Finance Discussion Paper, $\mathrm{n}^{\circ} 691$.

[3] A. MEDENOU, 2007 Note de cours d'économétrie des séries temporelles,

[4] BekAert, G. HARVeY, C.R. et Ng, A. (2003), "Market integration and contagion", NBER Working Paper, N $^{\circ}$ 9510.

[5] BeLLALAH. M (2000), «Gestion des Risques dans un Cadre International» ouvrage publié avec le concours du GREFI, université du Maine-Le Mains, ECONOMICA.

[6] Bellalah.M etAboura.S (2001), "The effect of Asymmetric Information and Transaction Costs on Asset Pricing: Theories and Tests ».

[7] BLAck.F, (1974), « international Capital Market Equilibrium with Investment Barriers», journal of financial Economics, 1, pp. 337-352.

[8] BORGY V.et MINGNON.V. (2006): taux d'intérêt et marches boursiers: une analyse empirique de l'intégration financière internationale.

[9] CoOPER I. ET Kaplanis E. (1994), «Home Bias in Equity Portfolios, Inflation Hedging, and International Capital Market Equilibrium», Review of Financial Studies, 7, pp. 45-60.

[10] De Melo, J. et Grether, (1997), Commerce international, théories et applications, De Boeck Université.

[11] Disdier, A.-C.etJ.-L., Mucchielli, (2002), «Biais domestique et concurrence des processus d'intégration dans les échanges de l'Europe du sud-est », Économie internationale 89-90 (2002). 
[12] Errunza V. et Padmanabhan P. (1992), "Tests of Integration, Mild Segmentation and Segmentation Hypothesis ", Journal of Banking and Finance, 16 , 949-472.

[13] FLAVIN T. ET WICKNES M. (2001), «Optimal International asset Allocation with Time-Varying Risk», Unpublished Manuscript, University of York.

[14] FrEnSH K. ET POTERBA J. (1991), «Investor Diversification and International Equity Markets», Americain Economic Review, 81, pp. 222-226.

[15] Gilmore, C. G. ET McMAnus, G. M. (2002), "International portfolio diversification: US and central European equity markets", Emerging Markets Review, Vol. 3, PP. 69-83.

[16] Gbetnkom,D.etD.,Avom, (2005), «Intégration par le marché : le cas de l'UEMOA », Régions et Développement $\mathrm{N}^{\circ} 22,2005$.

[17] GOYEAU D. LIONARD J ET PEPIN D.(2005) : « régime monétaire et processus d'intégration régionale des pays émergents ».

[18] Grubel H. (1968), "Internationally Diversified Portfolios: Welfare Gains and Capital Flows", American Economic Review, 58, pp. 1299-1314.

[19] Jean-Laurent.V, (2001), « Gestion de Portefeuille » ouvrage collectifs, DUNOD, pp. 67-84

[20] JESKE K. (2001), «Equity Home Bias: Can Information Cost Explain the Puzzle? », Federal Reserve Bank of Atlanta: Economic Review, 3, pp. 31-42.

[21] LEWIS K. (1999), «Trying to Explain Home Bias in Equities and Consumption », Journal of economic Literature, 6, pp.571-608.

[22] Laporte, B., (1996), “L'intégration monétaire avant l'intégration commerciale : le cas de l'Afrique de l'Ouest », Revue d'économie du développement.

[23] MaRkowizH. M, (1952), «Portfolios Selection », Journal of finance, vol. 7, n¹, pp. 77-91.

[24] MERTON R. (1987), «An Equilibrium Market Model with Incomplete Information», Journal of Finance, pp. 483544.

[25] Neaime S. (2002): "Liberalization and Financial Integration of MENA Stock Markets" Working Paper.

[26] Sendi I, Alloui C et Bellalah M. (2008), «The Equity Home Bais: Investors' Sentiments and Views », Accepted in Inter. Journal Of Behavioural accounting and Fiance..

[27] SolNIK B., (1974), «An Equilibrium Model of the International Capital Market», Journal of economic theory, pp. 500-524.

[28] STRONG N. et XU X. (2002), «Understanding the Equity Home Bias: Evidence From Survey Data», Working Paper.

[29] STULZ R., (1981), “ A Model of International Asset Pricing», Journal of Financial Economies, n9, pp.383406.

[30] TESAR L. et WERNER M. (1995), «Home Bias and High Turnover», Journal of International Money and Finance, 14, pp. 467-492. 